\section{More on modified dynamics}

SIR - Lindley's balanced comment ${ }^{1}$ on the standing of 'modified dynamics' $(\mathrm{MOND})^{2}$ as an alternative to dark matter should bring the issue closer to the focus of attention. We would like to clarify a few points.

MOND is not just a modification of the inverse distance square law of gravity. There have been several attempts to explain flat rotation curves of spiral galaxies by modifying inverse square attraction beyond some critical length scale; the clear prediction of such a modification is that larger galaxies should possess larger discrepancies between the observable and newtonian dynamical mass, but this is not observed $^{3}$. The fact is that discrepancies tend to appear at low accelerations, not at large distances, and this is the principal empirical motivation for MOND. Here, there is one new constant, a universal critical acceleration $a_{0}\left(\approx 10^{-8}\right.$ $\mathrm{cm} \mathrm{s} \mathrm{s}^{-1}$ ) below which the law of attraction or of inertia takes on an unfamiliar but specific form. Because of this the theory, when viewed as a modification of gravity, not only involves a different distance dependence for attraction, but also a different dependence on the attracting mass.

We do not think the "abrupt transition from normal to acceleration dependent gravity" is an "untidy" aspect of the theory. The transition is not more abrupt than that from non-relativistic physics to relativistic physics, or from classical to quantum physics.

Lindley notes, on the positive side, the success of MOND in explaining flat rotation curves of galaxies without dark matter. But it is not just that MOND in some very general sense implies flat rotation curves; it predicts the exact form of the rotation curve for a specific galaxy from the observed distribution of stars and gas, in some cases more successfully than multi-parameter dark halo models ${ }^{4}$. In addition the theory predicts the observed form of the luminosityrotation velocity relationship for spiral galaxies, the Tully-Fisher law; the luminosity-velocity dispersion relation for elliptical galaxies, the Faber-Jackson law; and the existence of a critical maximum surface brightness for spirals and ellipticals, the Freeman and Fish laws.

1. Lindley, D. Nature 359, 583 (1992).

2. Milgrom, M. Astrophys. J. 270, 365-389 (1983)

3. Sanders, R. H. Astr. astrophys. Rev. 2, 1-28 (1990).

4. Begeman, K. G., Breoils, A. H. \& Sanders, R. H. Mon Not. R. astr. Soc. 249, 523-537 (1991).

5. Bekenstein, J. D. in Proc. 2nd Canadian Conf. General

Relativity and Relativistic Astrophysics (eds Coley, A.

et al.) 68-103 (World Scientific, Singapore, 1987).

6. Sanders, R. H. Mon. Not. R. astr. Soc. 241, 135-151 (1989).

NATURE · VOL $362 \cdot 4$ MARCH 1993

Beyond galaxies, it predicts the correct magnitude of the missing mass in clusters of galaxies and in the Virgo supercluster - all of this with one constant, $a_{0}$.

Lindley echoes a recurring complaint that MOND is ad hoc and at present has no basis in deeper theory. We agree that, by and large, the rationale for the idea is phenomenological; however, the near equality of the fundamental acceleration and $\mathrm{cH}_{0}$ points to a possibly profound connection with cosmology and renders the theory less ad hoc. There have been several attempts at deriving a relativistic extension of MOND $^{5}$ and, although these theories contain physical anomalies, they do

\title{
Use of silk in ancient Egypt
}

SIK - On examining hair samples of mummies in the scanning electron microscope we found a piece of tissue between the curls which had the characteristic appearance of silk (see figure). To show that the specimen was silk, we performed infrared studies using multiple

\section{IMAGE UNAVAILABLE FOR COPYRIGHT REASONS}

Scanning electron micrograph of the textile specimen found in mummy's curl. Scale bar, $400 \mu \mathrm{m}$. (Photo by R. Surenian.)

internal reflection, allowing nondestructive identification of the material ${ }^{1}$. The spectra clearly identified silk.

We performed amino-acid analysis of the sample according to the method in ref. 2 and obtained the typical spectrum of hydrolysed silk, with high glycine, serine and alanine peaks as originally described by Shimura ${ }^{3}$. To exclude the possibility that the silk specimen could have been added later to the mummy's hair we performed amino-acid racemization studies on the mummy's hydrolysed hair samples and on the hydrolysed silk specimen: proline racemization was used as the marker amino acid. We used an HPLC method for the separation of $L$ and $\mathrm{D}$ forms $\mathrm{s}^{4}$.

The $\mathrm{D} / \mathrm{L}$ racemization ratios from hair and silk were comparable, which excludes contamination of hair by the silk tissue in recent times. The mummy, a 30-50-year-old female, was found in demonstrate the possibility of reconciling MOND with standard Big Bang cosmology ${ }^{6}$. Of course MOND would gain considerable respectability by making some connection to more familiar physics, but its success on a phenomenological level cannot be ignored. It is because of this that the idea slowly begins to attract the serious consideration which it deserves.

\section{Mllgrom}

Department of Physics,

Weizmann Institute of Science,

76100 Rehovot, Israel

R. H. Sanders

Kapteyn Astronomical Institute, 9700 AV Groningen, The Netherlands

Thebes, Deir el Medina, at the burial ground of the king's workmen. Based on anthropological data, the mummification method, the burial ground and aminoacid racemization, the mummy can be assigned to the twenty-first dynasty.

The silk industry had its origin in China and the material probably first reached the Mediterranean countries via Persia. Silk was not used in Egypt until later; the earliest example that can be traced is of Ptolemaic date from Mostagedda, a woollen tunic with decorative stripes with a weft of white silk. Lucan, writing in the middle of the first century, described Cleopatra with "her white breasts resplendent through the Sidonian fabric, which, wrought in close texture by the skill of the Seres, the needle of the workmen of the Nile has separated and has loosened the wrap by stretching out the web".

A portion of a coloured silk fabric was found at Qustul, south of Abu Simbel, the exact date of which is not certain, though it is probably not older than the fourth century AD. From the fourth century AD onwards silk became more common in Egypt. Our work suggests that silk was used in Egypt as long ago as 1,000 years $\mathrm{BC}$, which would shed new light on ancient trading practices.

\section{G. Lubec}

\section{J. Holaubek}

C. Feidl

B. Lubec

\section{E. Strouhal}

Department of Paediatrics,

University of Vienna,

A 1090 Vienna,

\section{Austria}

1. Lubec, G. J. arch. Sci. 14, 113-120 (1987)

2. Kedenburg, C. P. Analyt. Biochem. 40, 35-44 (1971).

3. Shimura, K. Experientia 39, 455-461 (1983).

4. Kampel, D. Kupferschmidt, R. \& Lubec, G. Amino Acids (eds Lubec, G. \& Rosenthal, G. A.) 1005-1012 (Escom, Leiden, 1990). 